\title{
Genome-Wide Identification and Expression Analysis of the NRAMP Family in Melon
}

\author{
Lan Jin ${ }^{1,2}$, Bayaer Enhe ${ }^{2}$, Yufeng Zhuang ${ }^{2}$, Agula Hasi ${ }^{{ }^{*}}$ and Feng Gao ${ }^{2 *}$ \\ ${ }^{1}$ Key Laboratory of Herbage and Endemic Crop Biotechnology (Inner Mongolia University), Ministry of Education, College \\ of Life Sciences, Inner Mongolia University, Hohhot 010070, China \\ ${ }^{2}$ College of Life Science and Technology, Inner Mongolia Normal University, Hohhot 010022, China \\ *For correspondence: hasind@ sina.com; imgaofeng@163.com \\ Received 15 August 2020; Accepted 15 October 2020; Published 10 January 2021
}

\begin{abstract}
The NRAMPs (natural resistance-associated macrophage proteins) are evolutionarily conserved integral membrane proteins that transport a broad range of metal ions. A comprehensive analysis of NRAMP family genes has not been reported for melon (Cucumis melo). In this study, six CmNRAMP genes were identified from the melon genome. Analyses of gene structure, phylogeny, chromosome location and conserved motifs revealed two groups of CmNRAMPs: one group contained only CmNRAMP5, which had 12 conserved motifs like NRAMPs in Arabidopsis thaliana and rice (Oryza sativa). The other group contained five CmNRAMPs and all had shorter amino acid sequences compared with CmNRAMP5. Expression analyses suggested that CmNRAMP genes are expressed differently in response to treatments with different plant hormones, abiotic factors, and metals (iron, copper, and manganese). The most responsive gene was CmNRAMP5, which showed significant changes in its transcript levels in response to abscisic acid (ABA), indole acetic acid, cytokinin, methyl jasmonate, brassinosteroid, $\mathrm{Fe}$, and $\mathrm{Cu}$. The least responsive gene was $C m N A R M P 6$, which was only significantly responsive to ABA, Fe, and $\mathrm{Mn}$. These results indicate that $C m N R A M P$ genes function in numerous tissues and at different developmental stages. A protein-protein interaction analysis indicated that CmNRAMPs interact with proteins involved in transmembrane transport, superoxide dismutase activity, plant hormone signal transduction, signal transduction by protein phosphorylation, and nicotianamine synthase activity. This study provides valuable insights into the potential function of $C m N R A M P$ genes and their encoded products. (C) 2021 Friends Science Publishers
\end{abstract}

Keywords: Cucumis melo; Expression analysis; NRAMP gene family; Protein-protein interaction

\section{Introduction}

Natural resistance-associated macrophage proteins (NRAMPs) are a highly evolutionarily conserved family of integral membrane proteins that are widely distributed among diverse organisms, including bacteria, yeast, algae, plants and animals (Nevo and Nelson 2006). The first NRAMP gene, NRAMP1, was identified in mammals, and was found to be expressed in phagosomes of infected macrophages (Vidal et al. 1993). The NRAMPs participate in resistance to bacterial infection by transporting metal ions such as manganese $\left(\mathrm{Mn}^{2+}\right)$ and iron $\left(\mathrm{Fe}^{2+}\right)$ (Supek et al. 1996; Fleming et al. 1997). The wide distribution of NRAMPs among different species indicates the importance of their function. Subsequent studies have shown that NRAMPs exhibit functional divergence and broad substrate specificity in different species. Various members of the NRAMP family function as proton-coupled metal ion transporters to transport manganese $\left(\mathrm{Mn}^{2+}\right)$, iron $\left(\mathrm{Fe}^{2+}\right)$, zinc $\left(\mathrm{Zn}^{2+}\right)$, copper $\left(\mathrm{Cu}^{2+}\right)$, cadmium $\left(\mathrm{Cd}^{2+}\right)$, nickel $\left(\mathrm{Ni}^{2+}\right)$, cobalt
$\left(\mathrm{Co}^{2+}\right)$, and aluminum $\left(\mathrm{Al}^{3+}\right)$ (Colangelo and Guerinot 2006; Nevo and Nelson 2006; Xia et al. 2010; Sasaki et al. 2012; Xiong et al. 2012; Li et al. 2014). These proteins have been implicated in the uptake, translocation, intracellular transport, and detoxification of transition metals (Nevo and Nelson 2006). The transport of metal ions in plants plays important roles in plant growth, development, signal transduction, nutrition, and protection against heavy metal poisoning. However, most studies on NRAMPs have been conducted in yeast, and their exact physiological roles in plants are still poorly understood.

Melon (Cucumis melo L.) is an economically important and widely cultivated vegetable crop (Huang et al. 2016; Xiong et al. 2018) and is an ideal model for analyzing the development and ripening of fleshy fruits (Pech et al. 2008; Ezura and Owino 2008). However, great economic losses are caused by biological and abiotic stresses such as diseases and adverse environmental conditions. In our previous study using suppression subtractive hybridization (SSH) analyses, we found that an expressed sequence tag

To cite this paper: Jin L, B Enhe, Y Zhuang, A Hasi, F Gao (2021). Genome-wide identification and expression analysis of the NRAMP family in melon. Intl J Agric Biol 25:327-337 
(EST) (MELO3C019215) encoding a NRAMP was differentially expressed during the ethylene climacteric burst in melon fruit (Gao et al. 2013).

In this work, the CmNRAMP5 gene fragment was used to identify all $N R A M P$-like gene sequences in the melon genome. Then, the expression patterns of CmNRAMP genes in different tissues at different stages, and in response to different hormones, stress, and metal ions were determined. Finally, to further understand the potential functions of these proteins, a protein-protein interaction network was constructed.

\section{Materials and Methods}

\section{Identification of NRAMP family genes in melon}

All candidate CmNRAMP genes were derived from the melon genome database (Zheng et al. 2019) using BLASTp searches. The search query was the NRAMP EST isolated from SSH libraries in our previous study (Gao et al. 2013). The predicted amino acid sequences of the candidate proteins were searched to identify NRAMP domains using the Simple Modular Architecture Research Tool (SMART) (Letunic and Bork 2018). Only the sequences with a fulllength NRAMP domain were considered as CmNRAMPs and used for further analyses.

\section{Sequence alignment, phylogenetic analysis and conserved motif analysis}

Multiple sequence alignment of the identified CmNRAMP sequences was performed using Clustal W with default parameters and was adjusted manually. Phylogenetic trees were drawn with MEGA 5.10 software using the neighborjoining method, and the reliability of the obtained trees was assessed with a bootstrap value of 1000 (Tamura et al. 2011). Conserved motifs were identified using MEME v. 5.1 .1 (http://meme-suite.org/tools/meme) with the following parameters: maximum number of motifs: 12 , motif width $>6$ and <200 (Bailey et al. 2009). The functional interaction networks of proteins were constructed using tools at the STRING protein interaction database (http://string-db.org/) with the confidence parameter set at 0.15 and threshold set at 75 . The resulting interaction information was directly imported into Cytoscape software (3.7.2) for visual editing (Shannon et al. 2003; Szklarczyk et al. 2019).

\section{Promoter analysis and determination of chromosomal locations}

The cis-acting regulatory elements in the promoter of the NRAMP gene were identified using tools at the PlantCARE database (Lescot et al. 2002). The promoter region was defined as the $2-\mathrm{kb}$ sequence upstream of the transcription start site. Using the sequences of the melon genome and NRAMP genes, we used TBtools software (Chen et al. 2018) to map the NRAMP genes onto the chromosomes.

\section{Plant materials and treatments}

Melon (C. melo L. cultivar Hetao) plants were grown in the field and the mesocarp of fruit ( 0 to 50 days after pollination; DAP) and internal ethylene were collected as described previously (Gao et al. 2013). Other tissues, such as roots, stems, young leaves, and flowers were also collected from greenhouse-grown plants. To minimize the effects of endogenous hormones on CmNRAMP transcription, sterile young leaves were transferred to $250-\mathrm{mL}$ flasks containing $100 \mathrm{~mL}$ of $1 / 2$-strength Murashige and Skoog (MS) liquid medium containing the treatment substance. All plant materials were sampled in three replicates. The flasks were incubated on a rotary shaker with shaking (100 rpm) for $2 \mathrm{~h}$ at $30^{\circ} \mathrm{C}$. The treatments were indole acetic acid (IAA, 0, 0.4, 4, $40 \mu \mathrm{M})$, gibberellic acid $\left(\mathrm{GA}_{3}, 0,0.4,4,40 \mu \mathrm{M}\right)$, salicylic acid (SA, 0, 100, 500, $1000 \mu \mathrm{M}$ ) cytokinin (CTK, 6benzylaminopurine, 6-BAP, 0, 0.4, 4, $40 \mu \mathrm{M}$ ), abscisic acid (ABA, 0, 0.4, 4, $40 \mu \mathrm{M}$ ), brassinosteroid (BR, 0, 0.01, 0.05, $0.1 \mathrm{mg} / \mathrm{L})$, peroxide $\left(\mathrm{H}_{2} \mathrm{O}_{2}, 0,1 \times 10^{4}, 2 \times 10^{4}, 4 \times 10^{4} \mu \mathrm{M}\right)$, methyl jasmonate (MeJA, 0, 4.46, 44.6, $446 \mu \mathrm{M}$ ) and metal ions of $\mathrm{Fe}^{2+}(0,50,100,200 \mathrm{mg} / \mathrm{L}), \mathrm{Cu}^{2+}(0,50,100,200$ $\mathrm{mg} / \mathrm{L})$, and $\mathrm{Mn}^{2+}(0,0.272,1.36,6.8 \mathrm{mg} / \mathrm{L})$ at different concentrations. Leaves were cultured in basal medium without any additive as the control. At the end of the culture, all leaves were removed, blotted dried, frozen in liquid nitrogen and then stored at $-80^{\circ} \mathrm{C}$ until RNA analysis.

\section{RNA isolation and qRT-PCR analysis}

Total RNA was extracted from plant samples using RNAiso for polysaccharide-rich plant tissue (Takara, Otsu, Japan) as per manufacturer's instructions. All RNA extracts were analyzed by agarose gel electrophoresis and UV spectrophotometry.

The CINRAMP mRNA levels were measured by quantitative PCR (qPCR). The primers (Table 1) were designed using Primer Premier v. 5.0, avoiding the conserved regions of the NRAMP motif and focusing on intron regions to reduce potential DNA contamination. For each trial, nine independent experiments were conducted (each experiment had three biological replicates, and each sample was analyzed with three technical replicates). All relative -fold differences in expression were normalized to the transcript level of GAPDH.

First-strand cDNA was synthesized using the PrimeScript ${ }^{\circledR}$ RT reagent kit with gDNA eraser (Perfect Real Time; Takara) following the manufacturer's protocol. For cDNA synthesis, $0.5 \mu \mathrm{g}$ of total RNA from each sample was used as template in a $10-\mu \mathrm{L}$ reaction mixture. SYBR ${ }^{\circledR}$ Premix Ex Taq ${ }^{\text {TM }}$ II (Tli RNaseH Plus; Takara) was used for real-time RT-PCR, with $5 \mu \mathrm{M}$ of each primer, and the reactions were run on a Mastercycler® ep realplex (Eppendorf, Hamburg, Germany). Melting curves were generated immediately after the last cycle to exclude any influence of primer dimers. Cycle numbers at which the 
Identification and Characterization of NRAMP Family in Melon / Intl J Agric Biol, Vol 25, No 2, 2021

Table 1: Sequences of primers used in qRT-PCR

\begin{tabular}{lllll}
\hline Gene name & Gene ID & Forward primer sequence (5'-3') & Reverse primer sequence (5'-3') & Product size (bp) \\
\hline CmNramp5 & MELO3C019215 & CTTGACGGAGAAGGTTGTGGTAAT & TGGCCGAAAGCAACTGGATC & 248 \\
CmNramp6 & MELO3C026742 & GAGAAATGAAGGGAGGGAGGTT & GAAGAAGGTGGATTCGACAAGC & 99 \\
CmNramp1 & MELO3C023938 & GTAAAGCTGAGTACCCCAAGGC & CACCAAACAGGAATGCGGAAG & 136 \\
CmNramp2 & MELO3C000512 & ATCATTGGTGGGTCTTCTGGG & CCATCTTGGCCTTACTGCTTG & 121 \\
CmGAPDH & AB033600 & ATCATTCCTAGCAGCACTGG & TTGGCATCAAATATGCTTGACCTG & 278 \\
\hline
\end{tabular}

Table 2: The information including amino acid length, number of exons and introns, and chromosomal assignment of six $C m N R A M P$ and $C m E I N 2$ genes

\begin{tabular}{lllllll}
\hline Gene & Gene identification & Location & Chromosome (no.) & Protein length (no. of amino acids) & Exons (no.) & Introns (no.) \\
\hline CmNRAMP1 & MELO3C023938 & $24502353-24505332$ & 4 & 324 & 9 & 8 \\
CmNRAMP2 & MELO3C000512 & $15521339-15522187$ & 0 & 73 & 2 & 1 \\
CmNRAMP3 & MELO3C010638 & $7913869-7915404$ & 3 & 310 & 7 & 6 \\
CmNRAMP4 & MELO3C021848 & $6615332-6617251$ & 11 & 283 & 5 & 4 \\
CmNRAMP5 & MELO3C019215 & $9194803-9198277$ & 11 & 510 & 4 & 3 \\
CmNRAMP6 & MELO3C026742 & $25044787-25048409$ & 4 & 324 & 10 & 9 \\
CmEIN2 & MELO3C014230 & $29460142-29457492$ & 8 & 1291 & 7 & 6 \\
\hline
\end{tabular}

fluorescence passed the cycle threshold $(\mathrm{Ct})$ were analyzed, and relative expression was calculated by the $2^{-\Delta \Delta \mathrm{Ct}}$ method. The differences were analyzed using DPS (Data Processing System) software (Tang and Zhang 2013).

\section{Results}

\section{Gene identification and sequence analysis}

We mapped the chromosomal locations of the genes based on the location information shown in Table 2, using TBtools software (Fig. 1). CmNRAMP1 and CmNRAMP6 were located on chromosome 4, CmNRAMP4 and CmNRAMP5 were located on chromosome 11 and the rest were located on different chromosomes.

Next, we analyzed the phylogenetic relationships between melon NRAMP genes and their homologs in $A$. thaliana and $O$. sativa. The tree had two large groups, in which both monocots and dicots were distributed (Fig. 2, Table S1). One group contained OsNRAMP1/6/5/4/3, AtNRAMP1/6, CmNRAMP3/4, and CmNRAMP1/6. OsNRAMP6/5/4/3 and AtNRAMP1/6 are plasma membrane-localized proteins. In rice, OsNRAMP4 (NRAT1) functions as a transporter specific for trivalent $\mathrm{Al}^{3+}$ (Xia et al. 2010; Li et al. 2014); OsNRAMP5 plays a role in the uptake of $\mathrm{Mn}^{2+}, \mathrm{Fe}^{2+}$, and $\mathrm{Cd}^{2+}$ from the soil (Sasaki et al. 2012); OsNRAMP6 is involved in uptake of $\mathrm{Fe}^{2+}$ and $\mathrm{Mn}^{2+}$, and contributes to disease resistance (Peris-Peris et al. 2017); and OsNRAMP3 functions as a switch in response to environmental $\mathrm{Mn}^{2+}$ changes (Yamaji et al. 2013). In Arabidopsis, AtNRAMP1/6 mediates $\mathrm{Mn}^{2+}$ uptake and $\mathrm{Cd}^{2+}$ toxicity, respectively (Cailliatte et al. 2009, 2010). The other group contained CmNRAMP5, AtNRAMP2/3/4/5 and OsNRAMP2/7. AtNRAMP3/4 are functionally redundant, and transport $\mathrm{Fe}^{2+}$ and $\mathrm{Mn}^{2+}$ and the toxic metal ion $\mathrm{Cd}^{2+}$ (Lanquar et al. 2005). AtNRAMP2 transports $\mathrm{Mn}^{2+}$ via the trans-Golgi network to support reactions in photosynthesis and cellular redox homeostasis (Alejandro et al. 2017; Gao et al. 2018).

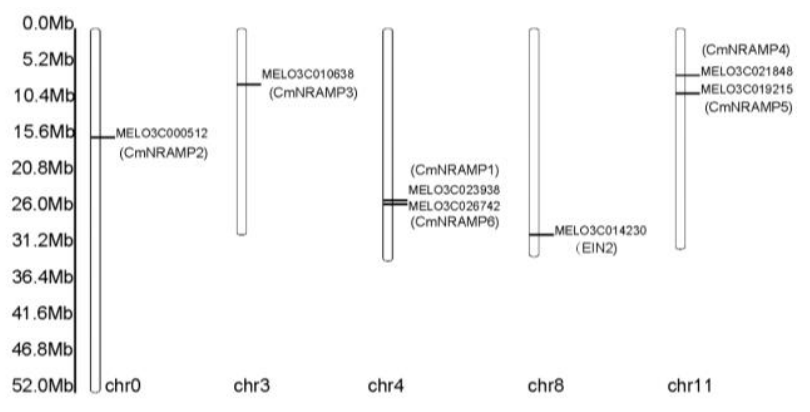

Fig. 1: Chromosomal locations of melon NRAMP genes Chr represents the chromosomes. The lengths of chromosomes of each NRAMP gene are displayed proportionately. Black lines on bars indicate the locations of NRAMP genes

To further examine the sequence features of plant NRAMPs, we conducted a comparative analysis of the conserved motifs among NRAMPs in melon, Arabidopsis, and rice. Twelve motifs were detected in NRAMP amino acid sequences, as revealed by analyses using the MEME program (Fig. S1). In general, NRAMPs that clustered in the same subgroups shared similar motif compositions (Fig. 2), indicating functional similarities among members of the same subgroup. Nearly all of the NRAMP members contained motifs $3,1,5,9,4$, and 6 , suggesting that these motifs are important for the functions of NRAMPs. Motif 12 was only present in members of subgroup II. All the members of this group except for AtNRAMP5 contained 12 motifs. Four NRAMPs in melon were shorter than their homologs in other plants. CmNRAMP3 and CmNRAMP4 were located on different chromosomes, and their encoded proteins lacked $\mathrm{N}$ and $\mathrm{C}$-terminal motifs and the $\mathrm{N}$-terminal motif, respectively. The proteins encoded by CmNRAMP6 and $C m N R A M P 1$ contained N-terminal motifs, but only CmNRAMP5 contained all motifs. The differences in motif distribution among NRAMPs suggested that the functions of their encoded proteins have diverged during evolution.

To better understand the potential regulation of CmNRAMP genes, we searched their promoter regions for 


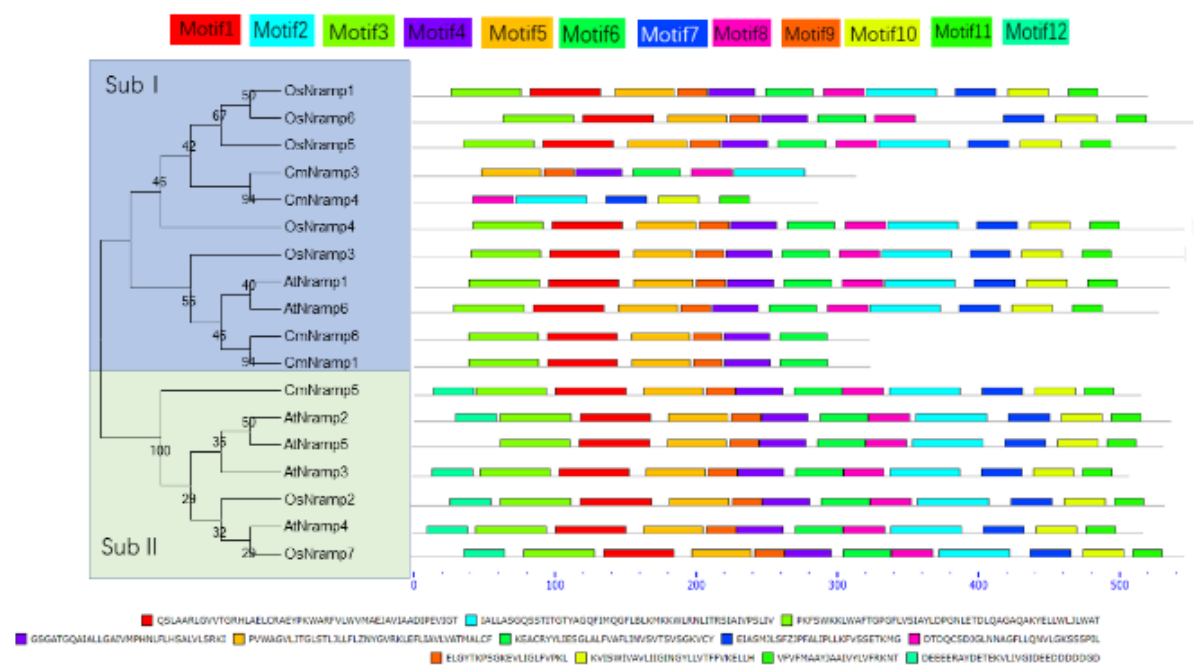

Fig. 2: Motif analysis of NRAMP proteins in C. melo, A. thaliana and O. sativa

A phylogenetic tree was constructed by MEGA5.10. Different subfamilies are marked with different color backgrounds. Motifs in the NRAMP proteins were elucidated by MEME. Different motifs are represented by different colored numbered boxes

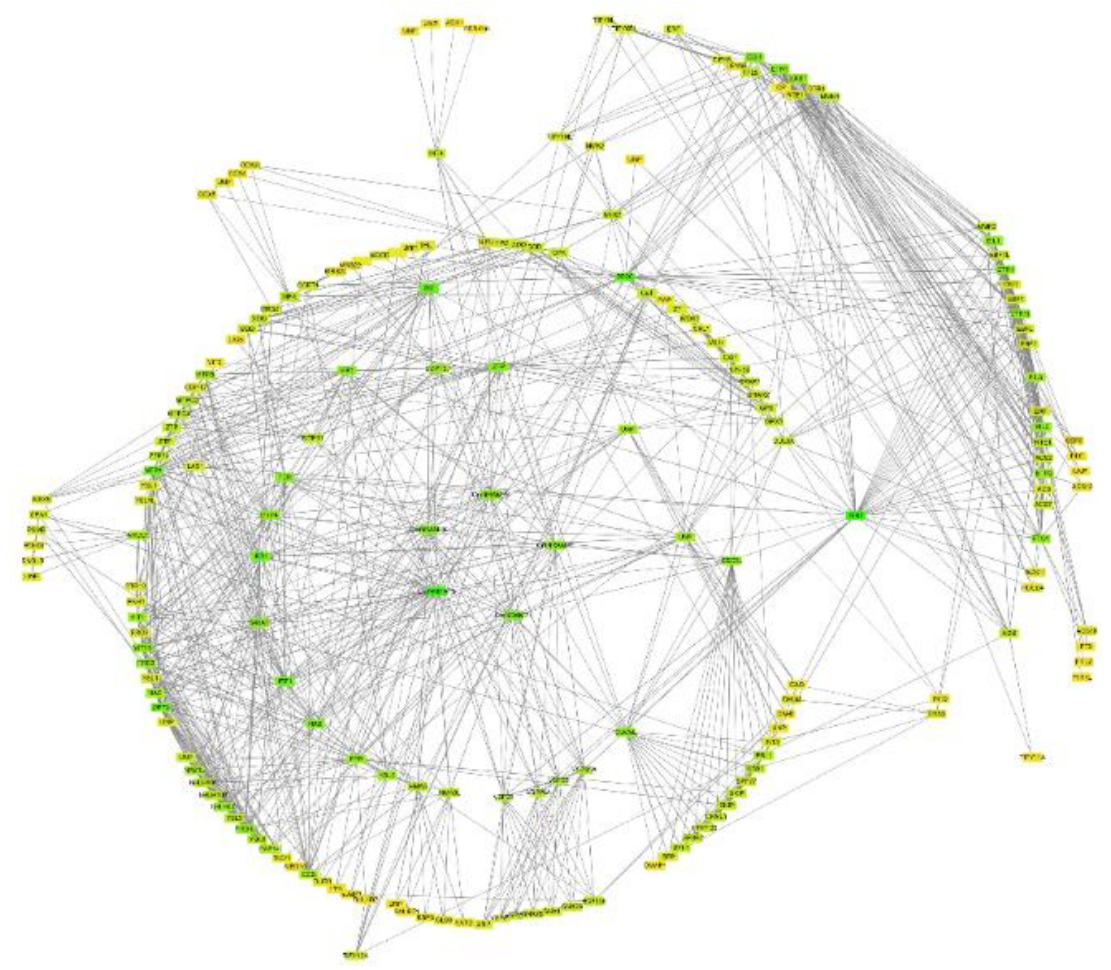

Fig. 3: Functional interaction networks of CmNRAMP proteins in melon according to STRING database and Cytoscape software 3.7.2 (IDARE)

cis-acting elements (Table S2). The promoter regions contained hormone response elements including methyl jasmonate (MeJA)-, gibberellin-, auxin- and SA-response elements; as well as low temperature-, drought-, and lightresponse elements. The results provided the basis for selecting different exogenous hormones to treat melon for analyses of $C m N R A M P$ gene expression patterns.

\section{Protein-protein interaction analysis}

To explore the interactions between NRAMPs and other proteins in melon, an interaction network was built. The CmNRAMP sequences were used as queries to obtain interacting protein information in the STRING database and the corresponding value was derived. The protein ID in the 


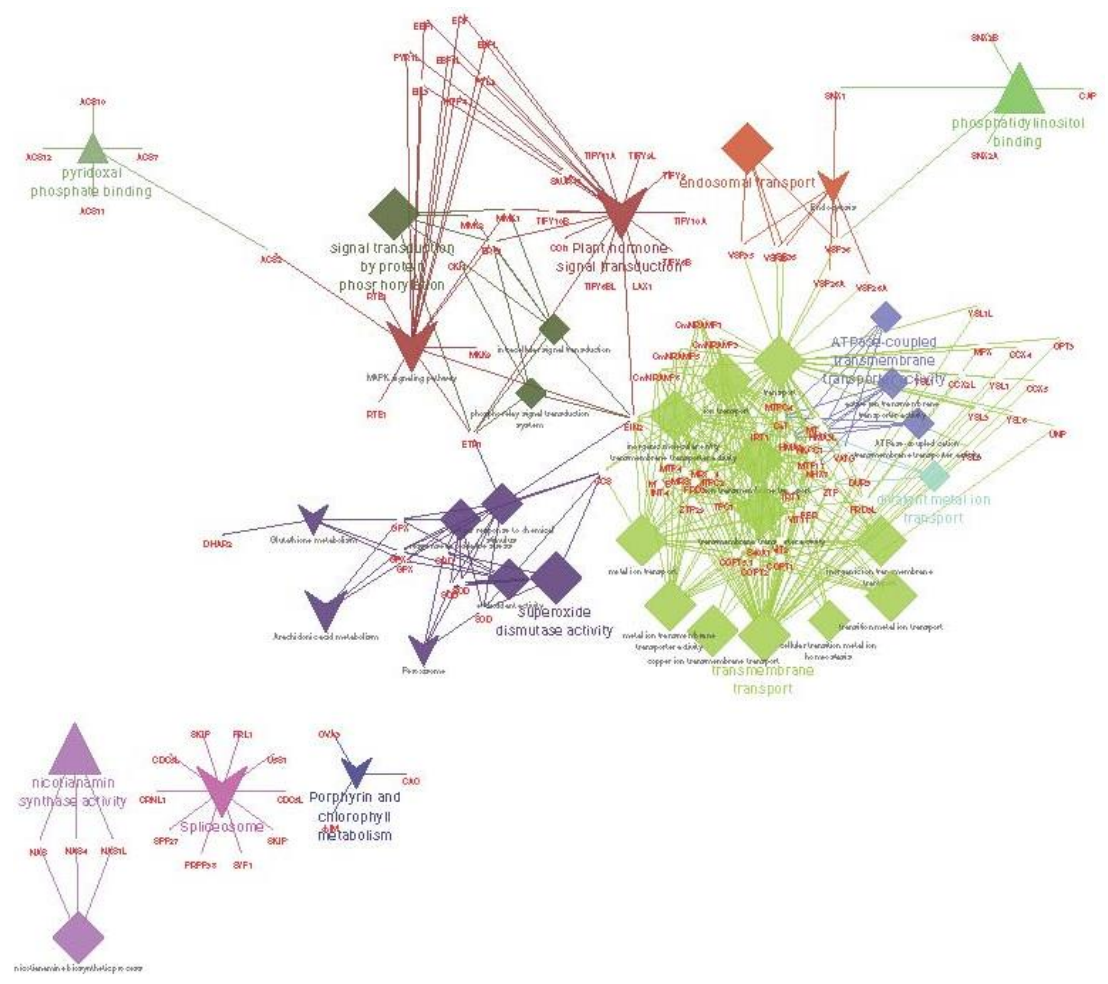

Fig. 4: Functional interaction networks of CmNRAMP proteins in melon according to STRING database and Cytoscape software 3.7 .2 (ClueGO)

The parallelogram indicates biological process, triangle indicates molecular function, and V-type indicates KEGG

STRING database was converted into the ID in the Uniprot database, and the network was visualized using Cytoscape.

A total of 192 proteins were obtained from the STRING database to construct the PPI, including proteins that participate in transmembrane transport, superoxide dismutase (SOD) activity, ATPase-coupled transmembrane transporter activity, signal transduction by protein phosphorylation, plant hormone signal transduction, nicotianamine synthase activity, endosomal transport, spliceosomes, porphyrin and chlorophyll metabolism, divalent metal ion transport, phosphatidylinositol binding, and pyridoxal phosphate binding (Fig. 3, Fig. 4, Fig. S2 and Table S3).

The NRAMPs are metal ion transporters, and the dynamic equilibrium of metal ions is known to affect diverse physiological responses. Our network analysis suggested that NRAMPs may play several roles in plant ethylene signal transduction. First, NRAMPs directly interact with VSP and CDC, which interact with EIN2. Second, NRAMPs have indirect interactions, with PP2C and other ethylene signal transduction proteins via their interaction with COP5.1 (all CmNRAMPs except CmNRAMP5) or CaT (all NRAMPs), or with EIN2 via their interactions with CaT (all CmNRAMPs).

The protein-protein interaction analysis also indicated that NRAMPs interact with the transcription factors FER and MT to affect SOD activity; and interact with IRT to affect the regulation of some transcription factors (bHLH47, bHLH 92, bHLH 100, bHLH 101). Interactions with HMA3 and HMA3L affect the regulation of GLP (involved in pathogen resistance), SAUR71 (involved in IAA signaling), and CCS (a copper chaperone for SOD). The interaction with CDC5L regulates spliceosomes via SYF1, SKIP, U5S1, and PRL1. Only CmNRAMP5 from the NRAMP family may directly interact with $\mathrm{NKCC} 1$ for regulation of SPA1, PSMB, PDH51, and DAGLB.

\section{Expression analyses}

Only four members of the gene family were annotated in the fruit transcriptome data (unpublished data). The qRT-PCR analyses confirmed that the other two family members were expressed at low levels or not expressed in the leaves. Therefore, the expression patterns of only four CmNRAMP genes were analyzed in this study.

Expression profiles of CmNRAMP genes in different tissues: The expression patterns of CmNRAMP genes in different tissues (roots, stem, leaves, and flowers) or fruit at different developmental stages (every 5 days from 0 to 50 DAP) were analyzed by qRT-PCR to explore their potential functions during the vegetative and reproductive development of melon.

The four analyzed CmNRAMP genes were detected in all tissues and showed variable transcript levels (Fig. 

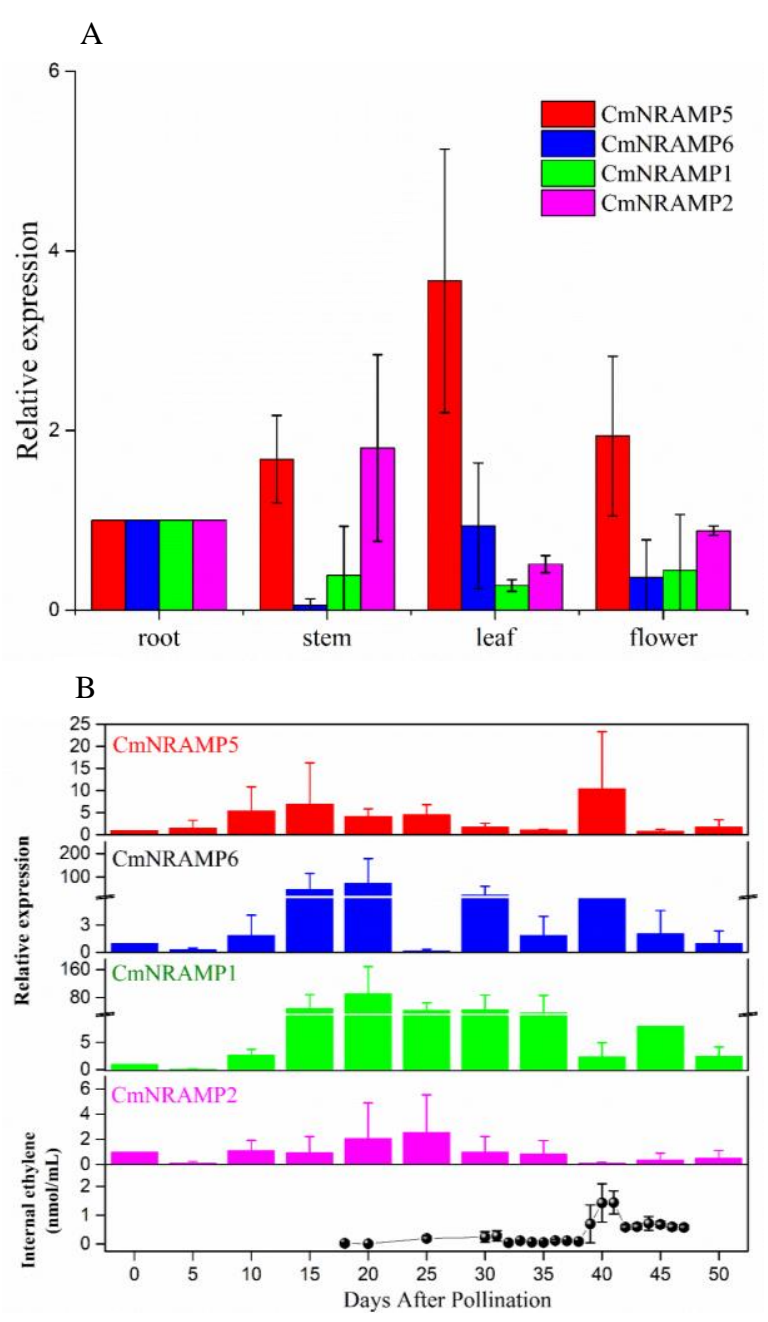

Fig. 5: Expression analysis of selected melon NRAMP genes in (A) different tissues and (B) fruit development stages using quantitative reverse-transcription polymerase chain reaction

5A). The transcript levels of CmNRAMP 2, 5, and 6 were the highest in the stem, leaves, and roots, respectively, and their transcript levels in these three tissues were not significantly different. CmNRAMP1 was abundantly expressed in the roots, and at significantly different levels among other tissues.

CmNRAMP genes showed high transcript levels during the fruit development stage (15-25 DAP), while the transcript level of CmNRAMP5 peaked at the same time as the climacteric peak of ethylene production (Fig. 5B). This result indicated that the expression of CmNRAMPs is at least partially ethylene-dependent or might be upstream regulator of ethylene.

Expression of CmNRAMP genes under different hormone treatments: We determined the changes in the transcript levels of CmNRAMP genes in response to treatments with ABA, CTK (6-BAP), BR, IAA, SA, MeJA, and GA at a range of concentrations. The transcript levels were quantified by qRT-PCR (Fig. 6).

Compared with the control group $(0 \mu \mathrm{mol} / \mathrm{L})$, the groups treated with $\mathrm{ABA}$ showed significantly decreased transcript levels of CmNRAMP1, 5, and 6. The transcript level of CmNRAMP2 increased and then decreased with increasing $\mathrm{ABA}$ concentrations.

Compared with the control, the groups treated with CTK tended to show decreased transcript levels of CmNRAMP1, 5, and 6 while that of CmNRAMP2 decreased, increased, and then decreased with increasing CTK concentrations. The results indicated that the gene transcript levels were significantly different between the control and the $40 \mu \mathrm{mol} / \mathrm{L}$ CTK treatment, but not among the 0.4 $\mu \mathrm{mol} / \mathrm{L}, 4 \mu \mathrm{mol} / \mathrm{L}$ and $40 \mu \mathrm{mol} / \mathrm{L}$ treatments. There was no significant difference in transcript levels among CmNRAMP1, 2 and 6 in each CTK treatment.

In the BR treatment, the transcript level of CmNRAMPI increased then decreased, those of CmNRAMP2 and 6 increased, and that of CmNRAMP5 decreased, compared with their respective levels in the control. The results indicated that there was no significant difference in transcript levels among CmNRAMP1, 2, and 6 at each BR concentration. The transcript level of CmNRAMP5 showed the largest decrease in the $0.05 \mathrm{mg} / \mathrm{L}$ BR treatment, to 0.1 times that in the control group. The results indicated that the transcript level of CmNRAMP5 differed significantly between the control and the $0.1 \mathrm{mg} / \mathrm{L}$ BR treatment, and between the control and the $4 \mathrm{~mol} / \mathrm{L} \mathrm{BR}$ treatment (extremely significant difference), but did not differ significantly among the $0.01 \mathrm{mg} / \mathrm{L}, 0.05 \mathrm{mg} / \mathrm{L}$ and $0.1 \mathrm{mg} / \mathrm{L}$ BR treatments.

In the IAA treatment, the transcript levels of the four genes decreased then increased with increasing IAA concentrations. The results indicated that there was no significant difference in transcript levels among CmNRAMP1, 2, and 6. The transcript level of CmNRAMP5 in the 0.4 $\mu \mathrm{mol} / \mathrm{L}$ IAA treatment was only 0.31 times that in the control group, but was 12.41 times that in the control group in the $40 \mu \mathrm{mol} / \mathrm{L}$ IAA treatment. The results indicated that the expression level of CmNRAMP5 differed significantly between the $40 \mu \mathrm{mol} / \mathrm{L}$ and $4 \mu \mathrm{mol} / \mathrm{L}$ IAA treatments, and between the control and the $40 \mu \mathrm{mol} / \mathrm{L}$ IAA treatment (extremely significant difference). There was no significant difference in the CmNRAMP5 transcript levels among the control and the $0.4 \mu \mathrm{mol} / \mathrm{L}$ and $4 \mu \mathrm{mol} / \mathrm{L}$ IAA treatments.

Treatment with SA increased the transcript levels of CmNRAMP1, 2, 5, and 6, compared with the control. The results indicated that the transcript levels of CmNRAMP6 in the $100 \mu \mathrm{mol} / \mathrm{L}$ and $500 \mu \mathrm{mol} / \mathrm{L}$ SA treatments were significantly different from that in the control. The transcript levels of CmNRAMP6 did not differ significantly among the other SA treatments.

In the MeJA treatments, the transcript levels of the four genes increased, decreased and then increased. CmNRAMP2 and 5 had the highest transcript levels in the $4.46 \mu \mathrm{mol} / \mathrm{L}$ treatment, which were 10.64 and 21.61 times that in the 
A

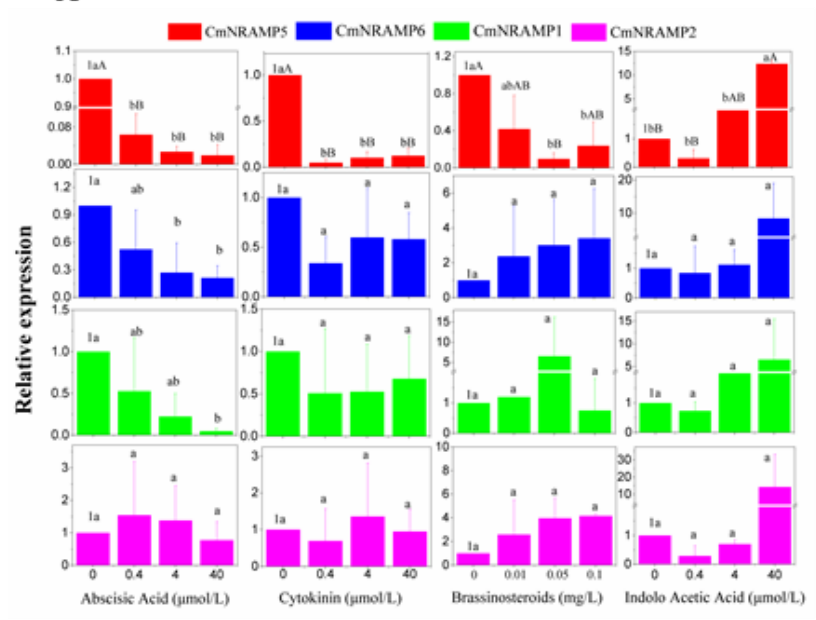

B

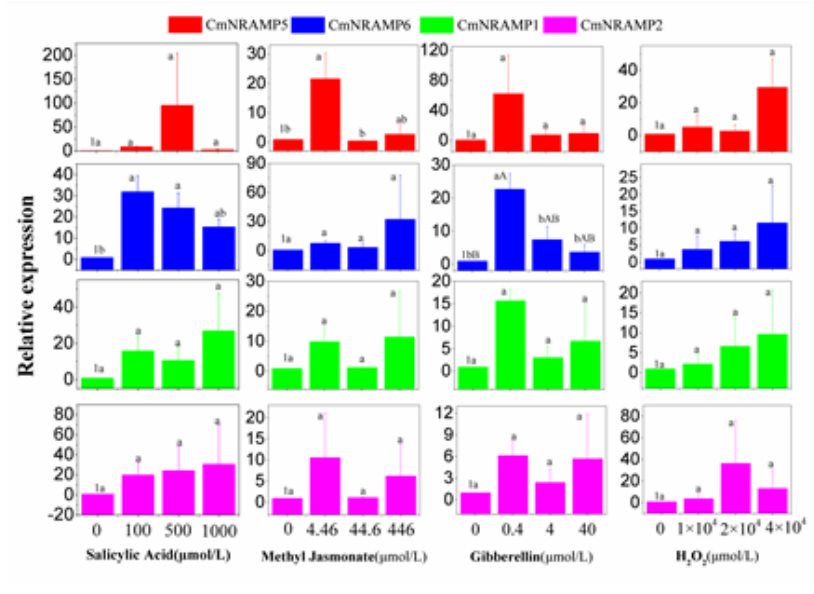

Fig. 6: Expression analysis of selected melon NRAMP genes in different hormones and stress treatment using quantitative reversetranscription polymerase chain reaction $(\mathbf{A}$ and $\mathbf{B})$

control, respectively. The transcript level of CmNRAMP5 in the $4.46 \mu \mathrm{mol} / \mathrm{L} \mathrm{MeJA}$ treatment was significantly different from those in the control and $44.6 \mu \mathrm{mol} / \mathrm{L} \mathrm{MeJA}$.

In the GA treatments, the transcript levels of the four genes were highest in the $0.4 \mu \mathrm{mol} / \mathrm{L} \mathrm{GA}$ treatment. The CmNRAMP6 transcript levels were significantly different between the $0.4 \mu \mathrm{mol} / \mathrm{L}$ and $4 \mu \mathrm{mol} / \mathrm{L}$ GA treatments, and extremely significantly different between the $0.4 \mu \mathrm{mol} / \mathrm{L}$ GA treatment and the control.

Expression of CmNRAMP genes in response to $\mathrm{H}_{2} \mathrm{O}_{2}$ : Treatment with $\mathrm{H}_{2} \mathrm{O}_{2}$ increased the transcript levels of all CmNRAMP genes, compared with the control. There was no significant difference in transcript levels among the four genes (Fig. 6).

Expression of CmNRAMP genes under different metal treatments: We determined changes in the transcript levels of CmNRAMP genes in melon roots in response to treatments with $\mathrm{Mn}^{2+}, \mathrm{Cu}^{2+}$, and $\mathrm{Fe}^{2+}$ using qRT-PCR analyses (Fig. 7).

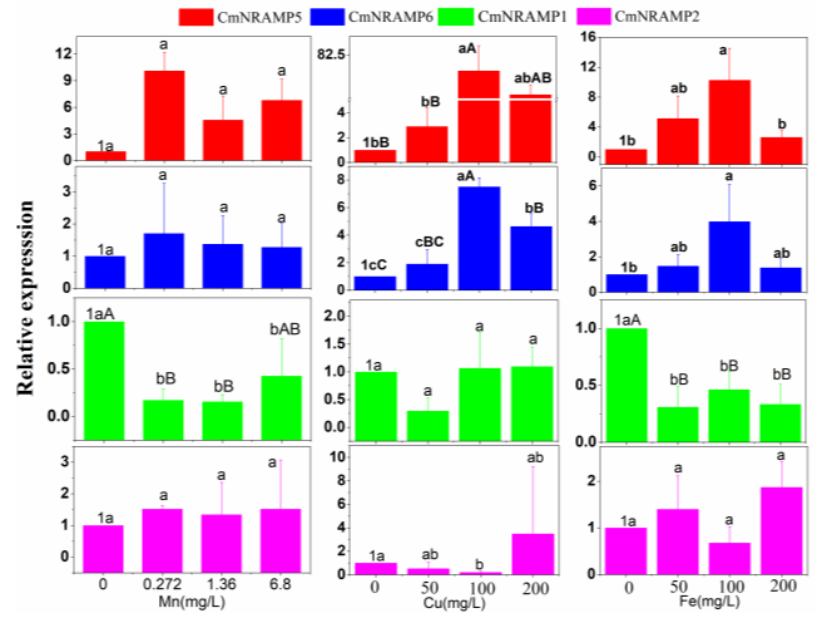

Fig. 7: Expression analysis of selected melon $N R A M P$ genes in three heavy metal treatment using quantitative reversetranscription polymerase chain reaction

Treatments with $\mathrm{Mn}^{2+}$ increased the transcript levels of CmNRAMP5 and 6, with both showing peak levels in the $0.272 \mathrm{mg} / \mathrm{L} \mathrm{Mn}{ }^{2+}$ treatment. There was no significant difference in the transcript levels of $C m N R A M P 2,5$, and 6 among the $\mathrm{Mn}^{2+}$ treatments. CmNRAMP1 was downregulated by $\mathrm{Mn}^{2+}$ treatments, with the lowest transcript level in the $0.272 \mathrm{mg} / \mathrm{L} \mathrm{Mn}^{2+}$ treatment. The transcript levels of CmNRAMPI differed significantly between the control and the $\mathrm{Mn}^{2+}$ treatments.

Treatment with $\mathrm{Cu}^{2+}$ up-regulated CmNRAMP5 and 6, suggesting their encoded proteins have transporter activity in melon. Both genes were most strongly induced in the 100 $\mathrm{mg} / \mathrm{L} \mathrm{Cu}^{2+}$ treatment, and were significantly higher in that group than in the control and other $\mathrm{Cu}^{2+}$ treatments. CmNRAMP2 was down-regulated by $\mathrm{Cu}^{2+}$, with the lowest transcript level in the $100 \mathrm{mg} / \mathrm{L} \mathrm{Cu}^{2+}$ treatment, where it was significantly lower than that in the control. However, the transcript levels of CmNRAMP1 were similar among all the $\mathrm{Cu}^{2+}$ treatments.

Treatment with $\mathrm{Fe}^{2+}$ up-regulated CmNRAMP5 and 6, down-regulated CmNRAMP1, and did not affected CmNRAMP2. These results suggested that CmNRAMP5 and 6 may have transporter activity in melon. The upregulated genes were most highly induced by $\mathrm{Fe}^{2+}$ at 100 $\mathrm{mg} / \mathrm{L}$, and their transcript levels were significantly higher than those in the control. The down-regulation of CmNRAMP1 was approximately the same among all the $\mathrm{Fe}^{2+}$ treatments, and its transcript levels were significantly lower than those in the control.

\section{Discussion}

Fruit ripening is regulated by a great deal of stimuli, including light, water availability, plant nutrient status, temperature, and hormones (Gao et al. 2013; Huang et al. 
2016). Melon is an economically important and widely cultivated vegetable crop that provides nutrients in the daily diet of consumers (Bie et al. 2017). In plant cells, metal ions are involved in physiological and biochemical reactions, and these reactions are affected by the enrichment and transport of metal ions. Therefore, it is important to study the relationship between metal transporters and the amount of heavy metal residues that accumulate during fruit development. Previous studies have shown that members of the NRAMP carrier family participate in the maintenance of metal homeostasis in Arabidopsis, rice, soybean, Malus baccata, Malus xiaojinensis, peanut, and Brassica napus (Xiao et al. 2008; Xiong et al. 2012; Zha et al. 2014; Pan et al. 2015; Meng et al. 2017; Qin et al. 2017). However, no detailed information was available for this family of transporters in melon. Here, we used a bioinformatics approach to identify the members of NRAMP family in the C. melo genome and to determine their biological roles during melon development.

In this study, searches of the melon genome revealed six putative CmNRAMP genes. Phylogenetic analysis clustered all of their encoded proteins into two distinct subfamilies. The subcellular localization of proteins in the same subfamily was similar among Arabidopsis, rice, common bean, and soybean (Qin et al. 2017; Mani and Sankaranarayanan 2018; Ishida et al. 2018). Interestingly, all of the melon NRAMPs except CmNRAMP5 contained fewer motifs and had shorter amino acid sequences than their homologs in other plants. Only CmNRAMP5, which was in its own subfamily, had the 12 characteristic NRAMP motifs.

We detected cis-acting elements in the promoter regions of CmNRAMPs and monitored changes in gene transcript levels in response to various treatments. These analyses revealed several hormone-responsive elements in the CmNRAMPs promoters. In the expression analyses, all the CmNRAMPs were up-regulated by SA, especially CmNRAMP6. CmNRAMP1/5/6 were down-regulated by $\mathrm{ABA}$, and all the CmNRAMPs were up-regulated by $\mathrm{H}_{2} \mathrm{O}_{2}$. Treatment with IAA affected the transcript levels of CmNRAMP5, while treatment with GA affected the transcript levels of CmNRAMP6. CmNRAMP5 transcript levels were affected by treatments with CTK, MeJA, and BR.

There were significant changes in the transcript levels of CmNRAMP1/5/6 in response to $\mathrm{Fe}^{2+}$; of CmNRAMP2/5/6 in response to $\mathrm{Cu}^{2+}$; and of CmNRAMPl in response to $\mathrm{Mn}^{2+}$. The closest homologs of CmNRAMP5 in Arabidopsis are AtNramp3/4. The transcript levels of AtNRAMP4 were shown to be greatly increased by 24 and $72 \mathrm{~h}$ of exposure to excess $\mathrm{Cu}$ (Zlobin et al. 2015). Our results and those of other studies indicate that the CmNRAMPs play important roles in responses to plant hormones and in maintaining metal ion homeostasis in melon.

NRAMPs are known to transport divalent metal ions, and some of them show functional redundancy, such as
AtNRAMP3 and AtNRAMP4 (Lanquar et al. 2005). However, some members of the NRAMP family can transport other ions, such as trivalent $\mathrm{Al}^{3+}$ ( $\mathrm{Li}$ et al. 2014). Pleiotropy of the NRAMPs can be demonstrated through PPI network analyses, and is fundamental for understanding their function. The ClueGO results indicated that the largest category of proteins in the protein-protein interaction network was transmembrane transporters. Other highly represented proteins were related to SOD activity, signal transduction by protein phosphorylation (MKKs), plant hormone signal transduction (ethylene, auxin, $\mathrm{ABA}$ ), and spliceosomes (CDC5L) (Fig. 4).

The protein-protein interaction network included proteins involved in plant hormone signal transduction. Several metal ions, especially $\mathrm{Cu}^{2+}$ have a critical role in ethylene perception and ethylene signaling (Hirayama and Alonso 2000). Ethylene perception requires $\mathrm{Cu}^{2+}$ for binding to ethylene receptors. In addition, EIN2, which has an NRAMP-like motif in N-terminal, plays positive role in ethylene signaling, especially the phosphorylationdependent cleavage from endoplasmic reticulum and nuclear movement of the EIN2-CEND peptide (Qiao et al. 2012). In melon overexpressing CmNRAMP5, the peak in the internal ethylene concentration in fruits occurred earlier than in wild type (unpublished result). SAUR71 is expressed in the steles of young roots and hypocotyls, and is differentially expressed during stomatal formation (Qiu et al. 2013). LAX2 is an auxin-responsive and/or auxin-related gene, and is strongly expressed in the primary root cap, where it is involved in specification of the quiescent center in Arabidopsis (Saito et al. 2019). PYL proteins are ABA receptors in Arabidopsis, and ABA perception by PYR/PYLs plays a major role in the regulation of seed germination and establishment (Gonzalez-Guzman et al. 2012). PYL1 inhibits protein phosphatase-type 2C upon binding of pyrabactin, an ABA agonist, whereas PYL2 appears relatively insensitive to this compound (Yuan et al. 2010).

The CDC5L protein is a core component of the putative E3 ubiquitin ligase complex, which plays roles in pre-messenger RNA splicing and in the cellular response to DNA damage. Recent studies have described a new function for CDC5L in the regulation of the ATR-mediated cell-cycle checkpoint in response to genotoxic agents (Zhang et al. 2009). It has been reported that CDC5 is involved in the ABA-mediated flooding tolerance of soybean (Komatsu et al. 2013), and is responsible for cell division and expansion during the thickening of the taproot in radish (Xie et al. 2018).

AtVPS35, which is localized in the pre-vacuolar compartment and immunoprecipitates with VPS29, is involved in sorting proteins to protein storage vacuoles in seeds, possibly by recycling vacuolar sorting receptor from the prevacuolar compartment to the Golgi complex. It is also involved in plant growth and senescence in vegetative organs (Yamazaki et al. 2008). AtVPS29 plays an important 
role in the trafficking of soluble proteins to the lytic vacuole from the trans-Golgi network to the prevacuolar compartment (Kang et al. 2012). The retromer components VPS35A and VPS29 are essential for normal prevacuolar compartment morphology and normal trafficking of plasma membrane proteins in plants (Nodzyński et al. 2013). A recent study showed that ZmVPS29 is involved in auxin accumulation during early kernel development in maize (Chen et al. 2020). An evolutionarily conserved VPS26 protein (VPS26C; At1G48550) functions in a complex with VPS35A and VPS29, which are necessary for root hair growth in Arabidopsis (Jha et al. 2018).

Other proteins identified in the protein-protein interaction network are known to have functions in transporting, binding, chelating, and chaperoning metal ions. These proteins had close homologs in Arabidopsis such as: (1) metal transporters (COPT5, YSL2); (2) enzymes involved in metal chelator synthesis (NAS1, and NAS2); (3) metal-binding proteins, including metallothionein (MT2); (4) metallochaperones (CCS); (Zlobin et al. 2015) and (5) metal tolerance proteins (MTPC2, MTPC4, MTP4, MTP11).

In previous studies on Arabidopsis, elevated $\mathrm{Cu}$ concentrations induced $C C S$ and YSL2 expression and inhibited NAS2 expression in roots, and induced $C C S$ and NRAMP4 expression in leaves. In canola, $\mathrm{Fe}$ and $\mathrm{Mn}$ contents in leaves were significantly decreased when plants were treated with $\mathrm{Cu}$ at high concentrations. The AtNRAMP3, AtNRAMP4 double mutant contained fewer functional photosystem II complexes, indicating that NRAMP transporters play important roles in photosystem II formation (Lanquar et al. 2010). Some proteins are known to interact with porphyrin and chlorophyll metabolism. Among them, $\mathrm{CAO}$, which is located on the inner envelope and thylakoid membranes of chloroplasts in Arabidopsis and barley, was able to catalyze the conversion of chlorophyllide a to chlorophyllide $\mathrm{b}$ in vitro (Reinbothe et al. 2006). Genetic studies have shown that ChlM is critical for chlorophyll biosynthesis and chloroplast development in tobacco and Arabidopsis (Alawady and Grimm 2005; Pontier et al. 2007). Proteins with phosphatidylinositol binding activity include SNX1, whose expression is induced by salt. This protein has NO synthase-like activity and produces NO under salt stress, which plays a crucial role in the development of salt tolerance (Li et al. 2018)

Under $\mathrm{Fe}$ deficiency, exogenous $\mathrm{NaCl}$ was shown to promote the reutilization of cell wall $\mathrm{Fe}$, and participate in the translocation of $\mathrm{Fe}$ from roots to shoots in Arabidopsis, partially because of its effects on ABA content. This was associated with the up-regulation of genes encoding proteins related to the long-distance transport of $\mathrm{Fe}$, such as NAS1 (Nicotianamine Synthase1), YSL2 (Yellow Stripe-Like) and FRD3 (Ferric Reductase Defective3) (Zhu et al. 2017).

HMA3 encodes heavy metal ATPase 3, which is responsible for cadmium (Cd) detoxification. In Sedum plumbizincicola, SpHMA3 was found to be highly expressed in shoots and its encoded protein was localized to the tonoplast (Liu et al. 2017). Overexpression of OsHMA3 increased $\mathrm{Cd}$ tolerance, and the plants produced rice grains with almost no $\mathrm{Cd}$, also had little effect on grain yield or on the concentrations of $\mathrm{Zn}, \mathrm{Fe}, \mathrm{Cu}$, and $\mathrm{Mn}$ (Lu et al. 2019).

A previous transcriptome study suggested that oxidative stress and protein denaturation are important contributors to arsenic (As) and Cd toxicity (Verbruggen $e t$ al. 2009). Phosphate (P) fertilizers are widely used in modern agriculture to improve crop growth. A higher $\mathrm{P}$ supply increases As and Cd uptake in shoots and roots, and excess heavy metals increase oxidative damage mediated by reactive oxygen species (ROS). The ROS are generated as a by-product of physiological reactions such as electron flow in chloroplasts and mitochondria and some redox reactions. The first line of defense against ROS is SODs, a group of metalloenzymes that include $\mathrm{Cu} / \mathrm{Zn} \mathrm{SOD}, \mathrm{Mn} \mathrm{SOD}$, and $\mathrm{Fe}$ SOD (Ozturk et al. 2010; Kung et al. 2014). Exposure to Cd was shown to increase the expression of $S O D$ genes in rice, but SOD expression in shoots and roots was found to decrease when rice plants were exposed to $\mathrm{Cd}$ without a phosphorus $(\mathrm{P})$ supply, resulting in oxidative damage (Wang et al. 2015). Other studies have shown that $\mathrm{P}$ deficiency affects $\mathrm{Fe}$ storage, as indicated by the accumulation of $\mathrm{Fe}$ associated with ferritin in chloroplasts. Inside chloroplasts, the expression of the ferritin gene, AtFerl, is regulated by the phosphate starvation response transcription factor AtPHR1, but this transcription factor does not affect the transcription of ITRl, which encodes a protein involved in Fe-uptake (Hirsch et al. 2006; Bournier et al. 2013). In soybean, most NRAMP genes displayed contrasting responses to $\mathrm{Fe}$ and sulfur deficiencies (Qin et al. 2017). The ATX1-like domain at the $\mathrm{N}$ terminus is essential for the $\mathrm{Cu}$ chaperone function of AtCCS in planta; this protein is essential for the integration of $\mathrm{Cu}$ into $\mathrm{Cu} / \mathrm{Zn} \mathrm{SOD}$ (Chu et al. 2005).

\section{Conclusion}

In this study, we identified the members of the NRAMP gene family in melon. Our results showed that only CmNRAMP5 has all the characteristic motifs, while the other five CmNRAMP have shorter amino acid sequences. The presence of particular cis-acting elements in the CmNRAMP promoter sequences and the expression patterns of the genes under different treatments, as verified by qPCR, indicate that the members of the NRMAP gene family respond to different plant hormones and metal ions. A protein-protein interaction analysis indicated that melon NRAMPs functions as metal ion transporters and interact with proteins involved in SOD activity, plant hormone signal transduction, signal transduction by protein phosphorylation, and nicotianamine synthase activity. This study provides insights into the diversity of melon NRAMPs, and provides baseline data for further comprehensive and in-depth analyses of their functions. 


\section{Acknowledgement}

We acknowledge the financial support of the National Natural Science Foundation of China (No.31660577), Natural Science Foundation of Inner Mongolia Autonomous Regin (2020MS03013) and Program for Young Talents of Science and Technology in Universities of Inner Mongolia Autonomous Region (No. NJYT-19B19). The English text of a draft of this manuscript was edited by Jennifer Smith, PhD, from Liwen Bianji, Edanz Group China.

\section{Author Contributions}

Agula Hasi and Feng Gao conceived and designed the experiments; Yufeng Zhuang and Lan Jin performed the experiments; Bayaer Enhe analyzed the data; Lan Jin wrote the paper.

\section{References}

Alawady AE, B Grimm (2005). Tobacco Mg protoporphyrin IX methyltransferase is involved in inverse activation of $\mathrm{Mg}$ porphyrin and protoheme synthesis. Plant J 41:282-290

Alejandro S, R Cailliatte, C Alcon, L Dirick, F Domergue, D Correia, L Castaings, JF Briat, S Mari, C Curie (2017). Intracellular distribution of manganese by the trans-Golgi network transporter NRAMP2 is critical for photosynthesis and cellular redox homeostasis. Plant Cell 29:3068-3084

Bailey TL, M Bodén, FA Buske, M Frith, CE Grant, L Clementi, J Ren, WW Li, WS Noble (2009). MEME SUITE: tools for motif discovery and searching. Nucl Acids Res 37:202-208

Bie Z, MA Nawaz, Y Huang, JM Lee, G Colla (2017). Introduction to vegetable grafting. In: Vegetable Grafting. Principles and Practices, pp: 1-21. Colla G, FP Alfocea, D Schwarz (Eds.). CABI Publishing, Wallingford, UK

Bournier M, N Tissot, S Mari, J Boucherez, E Lacombe, JF Briat, F Gaymard (2013). Arabidopsis ferritin 1 (AtFerl) gene regulation by the phosphate starvation response 1 (AtPHR1) transcription factor reveals a direct molecular link between iron and phosphate homeostasis. J Biol Chem 288:22670-22681

Cailliatte R, A Schikora, JF Briat, S Mari, C Curie (2010). High-affinity manganese uptake by the metal transporter NRAMP1 is essential for Arabidopsis growth in low manganese conditions. Plant Cell 22:904-917

Cailliatte R, B Lapeyre, JF Briat, S Mari, C Curie (2009). The NRAMP6 metal transporter contributes to cadmium toxicity. Biochem $J$ 422:217-228

Chen C, H Chen, Y He, R Xia (2018). TBtools, a toolkit for biologists integrating various HTS-data handling tools with a user-friendly interface. bioRxiv 2018; Article 289660

Chen L, YX Li, C Li, Y Shi, Y Song, D Zhang, H Wang, Y Li, T Wang (2020). The retromer protein ZmVPS29 regulates maize kernel morphology likely through an auxin-dependent process(es). Plant Biotechnol J 18:1004-1014

Chu CC, WC Lee, WY Guo, SM Pan, LJ Chen, H Li, TL Jinn (2005). A copper chaperone for superoxide dismutase that confers three types of copper/zinc superoxide dismutase activity in Arabidopsis. Plant Physiol 139:425-436

Colangelo EP, ML Guerinot (2006). Put the metal to the petal: Metal uptake and transport throughout plants. Curr Opin Plant Biol 9:322-330

Ezura H, WO Owino (2008). Melon, an alternative model plant for elucidating fruit ripening. Plant Sci 175:121-129
Fleming MD, CC Trenor, MA Su, D Foernzler, DR Beier, WF Dietrich, NC Andrews (1997). Microcytic anaemia mice have a mutation in Nramp2, a candidate iron transporter gene. Nat Genet 16:383-386

GaoF, Y Niu, J Hao, R Bade, L Zhang, A Hasi (2013). Identification of genes differentially expressed during ethylene climacteric of melon fruit by suppression subtractive hybridization. J Integr Agric 12:1431-1440

Gao H, W Xie, C Yang, J Xu, J Li, H Wang, X Chen, CF Huang (2018). $N R A M P 2$, a trans-Golgi network-localized manganese transporter, is required for Arabidopsis root growth under manganese deficiency. New Phytol 217:179-193

Gonzalez-Guzman M, GA Pizzio, R Antoni, F Vera-Sirera, E Merilo, GW Bassel, MA Fernández, MJ Holdsworth, MA Perez-Amador, H Kollist, PL Rodriguez (2012). Arabidopsis PYR/PYL/RCAR receptors play a major role in quantitative regulation of stomatal aperture and transcriptional response to abscisic acid. Plant Cell 24:2483-2496

Hirayama T, JM Alonso (2000). Ethylene captures a metal! metal ions are involved in ethylene perception and signal transduction. Plant Cell Physiol 41:548-555

Hirsch J, E Marin, M Floriani, S Chiarenza, P Richaud, L Nussaume, MC Thibaud (2006). Phosphate deficiency promotes modification of iron distribution in Arabidopsis plants. Biochimie 88:1767-1771

Huang Y, W Li, L Zhao, T Shen, J Sun, H Chen, Q Kong, MA Nawaz, Z Bie (2016). Melon fruit sugar and amino acid contents are affected by fruit setting method under protected cultivation. Sci Hortic 214:288-294

Ishida JK, DGG Caldas, LR Oliveira, GC Frederici, LMP Leite, TS Mui (2018). Genome-wide characterization of the NRAMP gene family in Phaseolus vulgaris provides insights into functional implications during common bean development. Genet Mol Biol 41:820-833

Jha SG, ER Larson, J Humble, DS Domozych, DS Barrington, ML Tierney (2018). Vacuolar protein sorting 26C encodes an evolutionarily conserved large retromer subunit in eukaryotes that is important for root hair growth in Arabidopsis thaliana. Plant J 94:595-611

Kang H, SY Kim, K Song, EJ Sohn, Y Lee, DW Lee, I Hara-Nishimura, I Hwang (2012). Trafficking of vacuolar proteins: the crucial role of Arabidopsis vacuolar protein sorting 29 in recycling vacuolar sorting receptor. Plant Cell 24:5058-5073

Komatsu S, C Han, Y Nanjo, M Altaf-Un-Nahar, K Wang, D He, P Yang (2013). Label-free quantitative proteomic analysis of abscisic acid effect in early-stage soybean under flooding. $J$ Proteom Res 12:4769-4784

Kung CP, YR Wu, H Chuang (2014). Expression of a dye-decolorizing peroxidase results in hypersensitive response to cadmium stress through reducing the ROS signal in Arabidopsis. Environ Exp Bot 101:47-55

Lanquar V, MS Ramos, F Lelièvre, H Barbier-Brygoo, A Krieger-Liszkay, U Krämer, S Thomine (2010). Export of vacuolar manganese by AtNRAMP3 and AtNRAMP4 is required for optimal photosynthesis and growth under manganese deficiency. Plant Physiol 152:1986-1999

Lanquar V, F Lelièvre, S Bolte, C Hamès, C Alcon, D Neumann, G Vansuyt, C Curie, A Schröder, U Krämer, H Barbier-Brygoo, S Thomine (2005). Mobilization of vacuolar iron by AtNRAMP3 and AtNRAMP4 is essential for seed germination on low iron. EMBO J 24:4041-4051

Lescot M, P Déhais, G Thijs, K Marchal, Y Moreau, VVD Peer, P Rouzé, S Rombauts (2002). PlantCARE, a database of plant cis-acting regulatory elements and a portal to tools for in silico analysis of promoter sequences. Nucl Acids Res 30:325-327

Letunic I, P Bork (2018). 20 years of the SMART protein domain annotation resource. Nucl Acids Res 46:493-496

Li JY, J Liu, D Dong, X Jia, SR McCouch, LV Kochian (2014). Natural variation underlies alterations in Nramp aluminum transporter (NRAT1) expression and function that play a key role in rice aluminum tolerance. Proc Natl Acad Sci USA 111:6503-6508

Li TT, WC Liu, FF Wang, QB Ma, YT Lu, TT Yuan (2018). SORTING NEXIN 1 functions in plant salt stress tolerance through changes of NO accumulation by regulating NO synthase-like activity. Front Plant Sci 9; Article 1634 
Liu H, H Zhao, L Wu, A Liu, F J Zhao, W Xu (2017). Heavy metal ATPase 3 (HMA3) confers cadmium hypertoleranceon the cadmium/zinc hyperaccumulator Sedum plumbizincicola. New Phytol 215:687-698

LuC, L Zhang, Z Tang, X Huang, J Ma, F Zhao (2019). Producing cadmiumfree Indicarice by overexpressing OsHMA3. Environ Intl 126:619-626

Mani A, K Sankaranarayanan (2018). In silico analysis of natural resistanceassociated macrophage protein (NRAMP) family of transporters in rice. Protein J 37:237-247

Meng JG, XD Zhang, SK Tan, KX Zhao, ZM Yang (2017). Genome-wide identification of Cd-responsive NRAMP transporter genes and analyzing expression of NRAMP 1 mediated by miR167 in Brassica napus. Biometals 30:917-931

Nevo Y, N Nelson (2006). The NRAMP family of metal-ion transporters. Biochim Biophys Acta 1763:609-620

Nodzyński T, MI Feraru, S Hirsch, RD Rycke, C Niculaes, W Boerjan, JV Leene, GD Jaeger, S Vanneste, J Friml (2013). Retromer subunits VPS35A and VPS29 mediate prevacuolar compartment (PVC) function in Arabidopsis. Mol Plant 6:1849-1862

Ozturk F, F Duman, Z Leblebici, R Temizgul (2010). Arsenic accumulation and biological responses of watercress (Nasturtium officinale $\mathrm{R}$. Br.) exposed to arsenite. $J$ Exp Bot 69:167-174

Pan H, Y Wang, Q Zha, M Yuan, L Yin, T Wu, X Zhang, X Xu, Z Han (2015). Iron deficiency stress can induce MxNRAMP1 protein endocytosis in M. xiaojinensis. Gene 567:225-234

Pech JC, M Bouzayen, A Latchq (2008). Climacteric fruit ripening: ethylene-dependent and independent regulation of ripening pathways in melon fruit. Plant Sci 175:114-120

Peris-Peris C, A Serra-Cardona, F Sánchez-Sanuy, S Campo, J Ariño, BS Segundo (2017). Two NRAMP6 isoforms function as iron and manganese transporters and contribute to disease resistance in rice. molecular plant-microbe interactions. Mol Plant Microb 30:385-398

Pontier D, C Albrieux, J Joyard, T Lagrange, MA Block (2007). Knock-out of the magnesium protoporphyrin IX methyltransferase gene in Arabidopsis: effect on chloroplast development and on chloroplastto-nucleus signaling. J Biol Chem 282:2297-2304

Qiao H, Z Shen, S Carol Huang, RJ Schmitz, MA Urich, SP Briggs, JR Ecker (2012). Processing and subcellular trafficking of ER-tethered EIN2 control response to ethylene gas. Science 338:390-393

Qin L, P Han, L Chen, TC Walk, Y Li, X Hu, L Xie, H Liao, X Liao (2017). Genome-wideidentification and expression analysis of NRAMP family genes in soybean (Glycine max L.). Front Plant Sci 8; Article 1436

Qiu T, Y Chen, M Li, Y Kong, Y Zhu, N Han, H Bian, M Zhu, J Wang (2013). The tissue-specific and developmentally regulated expression patterns of the SAUR41 subfamily of small auxin up RNA genes: potential implications. Plant Signal Behav 8; Article e25283

Reinbothe C, S Bartsch, LL Eggink, JK Hoober, J Brusslan, R Andrade-Paz, J Monnet, S Reinbothe (2006). A role for chlorophyllide a oxygenase in the regulated import and stabilization of light-harvesting chlorophyll a/b proteins. Proc Natl Acad Sci USA 103:4777-4782

Saito T, P Opio, S Wang, K Ohkawa, S Kondo, T Maejima, H Ohara (2019). Association of auxin, cytokinin, abscisic acid, and plant peptide response genes during adventitious root formation in Marubakaido apple rootstock (Malus prunifolia Borkh. var. ringo Asami). Acta Physiol Plantarum 41; Article 41

Sasaki A, N Yamaji, K Yokosho, JF Ma (2012). Nramp5 is a major transporter responsible for manganese and cadmium uptake in rice. Plant Cell 24:2155-2167

Shannon P, A Markiel, O Ozier, NS Baliga, JT Wang, D Ramage, N Amin, B Schwikowski, T Ideker (2003). Cytoscape: A software environment for integrated models of biomolecular interaction networks. Genomic Res 13:2498-2504

Supek F, L Supekova, H Nelson, N Nelson (1996). A yeast manganese transporter related to the macrophage protein involved in conferring resistance to mycobacteria. Proc Natl Acad Sci USA 93:5105-5110

Szklarczyk D, AL Gable, D Lyon, A Junge, S Wyder, J Huerta-Cepas, M Simonovic, NT Doncheva, JH Morris, P Bork, LJ Jensen, C von Mering (2019). STRING v11: protein-protein association networks with increased coverage, supporting functional discovery in genomewide experimental datasets. Nucl Acids Res 47:607-613
Tamura K, D Peterson, N Peterson, G Stecher, M Nei, S Kumar (2011). MEGA5: Molecular evolutionary genetics analysis using maximum likelihood, evolutionary distance, and maximum parsimony methods. Mol Biol Evol 28:2731-2739

Tang QY, CX Zhang (2013). Data Processing System (DPS) software with experimental design, statistical analysis and data mining developed for use in entomological research. Ins Sci 20:254-260

Verbruggen N, C Hermans, H Schat (2009). Mechanisms to cope with arsenic or cadmium excess in plants. Curr Opin Plant Biol $12: 364-372$

Vidal SM, D Malo, K Vogan, E Skamene, P Gros (1993). Natural resistance to infection with intracellular parasites: isolation of a candidate for Bcg. Cell 73:469-485

Wang H, T Wang, I Ahmad (2015). Involvement of phosphate supplies in different transcriptional regulation pathway of Oryza sativa L.'s antioxidative system in response to arsenite and cadmium stress. Ecotoxicology 24:1259-1268

Xia J, N Yamaji, T Kasai, JF Ma (2010). Plasma membrane localized transporter for aluminum in rice. Proc Natl Acad Sci USA 107:18381-18385

Xiao H, L Yin, X Xu, T Li, Z Han (2008). The iron-regulated transporter, $M b N R A M P 1$, isolated from Malus baccata is involved in $\mathrm{Fe}, \mathrm{Mn}$ and Cd trafficking. Ann Bot 102:881-889

Xie Y, L Xu, Y Wang, L Fan, Y Chen, M Tang, X Luo, L Liu (2018). Comparative proteomic analysis provides insight into a complex regulatory network of taproot formation in radish (Raphanus sativus L.). Hortic Res 5:51-64

Xiong H, T Kobayashi, Y Kakei, T Senoura, M Nakazono, H Takahashi, H Nakanishi, H Shen, P Duan, X Guo, N Nishizawa, Y Zuo (2012). AhNRAMP1 iron transporter in involved in iron acquisition in peanut. $J$ Exp Bot 63:4437-4446

Xiong M, X Zhang, S Shabala, L Shabala, Y Chen, C Xiang, MA Nawaz, Z Bie, H Wu, H Yi, M Wu, Y Huang (2018). Evaluation of salt tolerance and contributing ionic mechanism in nine Hami melon landraces in Xinjiang, China. Sci Horitc 237:277-286

Yamaji N, A Sasaki, JX Xia, K Yokosho, JF Ma (2013). A node-based switch for preferential distribution of manganese in rice. Nat Commun 4; 2442

Yamazaki N, T Shimada, H Takahashi, K Tamura, M Kondo, M Nishimura, I Hara-Nishimura (2008). Arabidopsis VPS35, a retromer component, is required for vacuolar protein sorting and involved in plant growth and leaf senescence. Plant Cell Physiol 49:142-156

Yuan X, P Yin, Q Hao, C Yan, J Wang, N Yan (2010). Single amino acid alteration between valine and isoleucine determines the distinct pyrabactin selectivity by PYL1 and PYL2. J Biol Chem 285:28953-28958

Zha Q, Y Wang, XZ Zhang, ZH Han (2014). Both immanently high active iron contents and increased root ferrous uptake in response to low iron stress contribute to the iron deficiency tolerance in Malus xiaojinensis. Plant Sci 214:47-56

Zhang N, R Kaur, S Akhter, RJ Legerski (2009). Cdc5L interacts with ATR and is required for the S-phase cell-cycle checkpoint. EMBO Rep 10:1029-1035

Zheng Y, S Wu, Y Bai, H Sun, C Jiao, S Guo, ZK Hao, J Blanca, Z Zhang, S Huang, Y Xu, Y Weng, M Mazourek, UK Reddy, K Ando, JD McCreight, AA Schaffer, J Burger, Y Tadmor, N Katzir, X Tang, Y Liu, JJ Giovannoni, KS Ling, WP Wechter, A Levi, J Garcia-Mas, R Grumet, Z Fei (2019). Cucurbit Genomics Database (CuGenDB): a central portal for comparative and functional genomics of cucurbit crops. Nucl Acids Res 47:1128-1136

Zhu XF, Q Wu, L Zheng, RF Shen (2017). NaCl alleviates iron deficiency through facilitating root cell wall iron reutilization and its translocation to the shoot in Arabidopsis thaliana. Plant Soil 417:155-167

Zlobin IE, VP Kholodova, ZF Rakhmankulova, VV Kuznetsov (2015). Brassica napus responses to short-term excessive copper treatment with decrease of photosynthetic pigments, differential expression of heavy metal homeostasis genes including activation of gene NRAMP4 involved in photosystem II stabilization. Photosynth Res 125:141-150 